\title{
An Unusual Cause of Acute Pancreatitis
}

\author{
Jung Wan Choe \\ Department of Internal Medicine, Korea University College of Medicine, Seoul, Korea
}

Received October 25, 2021, Revised November 11, 2021, Accepted November 11, 2021

Corresponding author: Jung Wan Choe, E-mail: jwchoe@korea.ac.kr, https://orcid.org/0000-0003-0634-5141

QUESTION: A 68-year-old male underwent a distal pancreatectomy to treat T2N1M0 pancreatic tail cancer. After six weeks of surgery, the patient's general condition was good, and a total of six cycles of primary adjuvant chemotherapy with 5 -fluorouracil (5-FU) $\left(425 \mathrm{mg} / \mathrm{m}^{2}\right)$ and folic acid $\left(20 \mathrm{mg} / \mathrm{m}^{2}\right)$ every four weeks was planned. After the end of the third cycle of chemotherapy, the patient visited the emergency room with epigastric pain and mild fever. The blood tests revealed $635 \mathrm{IU} / \mathrm{L}$ amylase and $559 \mathrm{IU} / \mathrm{L}$ lipase. The other laboratory results were within the normal ranges. Abdominal computed tomography (CT) revealed fluid collection at the pancreatic resection margin with diffuse mesenteric and omental infiltration in the pericolic (hepatic flexure colon, transverse colon), perigastric, and periduodenal regions (Fig. 1).

There was no evidence of postoperative leakage of pancreatic juice, recurring pancreatic cancer, or gallstones. He had not taken any over-the-counter drugs and had no history of alcohol consumption or other risk factors commonly associated with pancreatitis. The diagnosis of acute pancreatitis was made without a definite etiology. During the next two weeks, he received conservative care and bowel rest with intravenous hydration. The complaint symptoms were diminished and pancreatic enzyme levels were also normalized. He was discharged from the hospital and planned to be admitted again on his next scheduled chemotherapy day. However, a recurrent pancreatitis episode occurred two days following the completion of the 5-FU infusion in the next (fourth) cycle. At that time, severe abdominal pain persisted, and the serum amylase and lipase levels had increased to 419 IU/L and 393 IU/L, respectively. A CT scan was performed and revealed that inflammatory infiltration into the mesentery and omentum had progressed more extensively than in the previous examination. Multiple large pseudocysts were observed around the pancreas (Fig. 2).

However, the cause of recurrent pancreatitis was not clearly determined in the CT scan or the following magnetic resonance imaging. All the possible common causes of acute pancreatitis were excluded. Moreover, considering the relationship between the time of administration of the chemotherapeutic agents and the recurrence of pancreatitis follow-

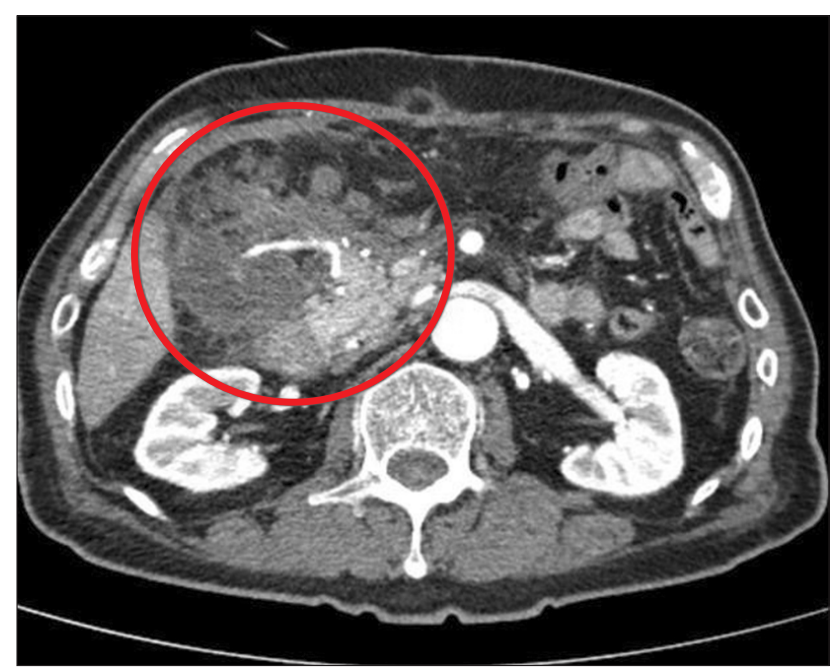

Fig. 1. Abdominal computed tomography image of first occurrence of pancreatitis. 


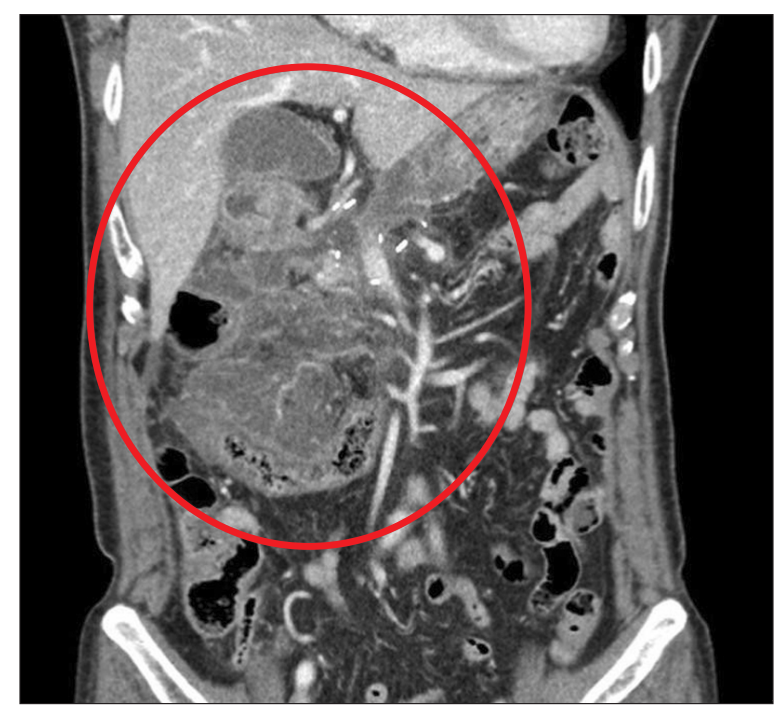

Fig. 2. Abdominal computed tomography image of recurrent pancreatitis.

ing re-administration, the possibility of drug-induced acute pancreatitis (DIP) from chemotherapeutic drug administration was high. After the second episode of pancreatitis, we decided to stop further adjuvant chemotherapy. Since then, the patient did not experience pancreatitis recurrence and no complications due to pancreatitis were observed. Currently, he is followed at an outpatient clinic with no evidence of pancreatic cancer recurrence for four years.

What is the diagnosis for this case?

ANSWER: Drug-induced acute pancreatitis.

REVIEW: Alcohol and gallstones are the common causes of acute pancreatitis, but it can also be caused by hypertriglyceridemia, hypercalcemia, pancreatic malformations including a pancreatic mass, trauma, infection, autoimmune disease, and drugs. Drug-induced acute pancreatitis is assumed to be associated with various drugs, accounting for about $0.1-2 \%$ of acute pancreatitis [1]. Compared to the Western countries, DIP has been considered a relatively rare disease in Korea, but recently several cases of DIP were reported in Korea, and the frequency of occurrence is steadily increasing [2]. However, DIP induced by 5 -FU has been reported very rarely as a case report, and there was only one case, which was confirmed by rechallenge with 5-FU [3].
The diagnosis of DIP can be confirmed 1) when other causes are excluded, 2) the consecutive relationship between the administration of a pancreatitis-inducing drug and the onset of acute pancreatitis is demonstrated, and 3) the recurrence of pancreatitis through re-administration is proved. The mechanism of the development of DIP is unclear but an immunologic reaction following drug use, a direct toxic effect with the accumulation of toxic metabolites, ischemia, intravascular thrombosis, and increased pancreatic juice viscosity have been suggested. The most common mechanism is an immunologic reaction. Acute pancreatitis occurs between one and six weeks of administration, regardless of the dose of the causative drug. Acute pancreatitis was shown to recur within 1-3 days when the drug was re-administered [4]. In contrast, acute pancreatitis caused by the accumulation of toxic metabolites causes pancreatic damage in a dosedependent manner and occurs several months after administration. DIP has no specific clinical and laboratory findings and is similar to other types of pancreatitis. Most DIP shows a better prognosis than pancreatitis caused by other causes because DIP improves upon stopping the drugs.

In conclusion, clinicians should always keep in mind that DIP can arise from chemotherapy in pancreatic cancer patients.

\section{CONFLICTS OF INTEREST}

No potential conflict of interest relevant to this article was reported.

\section{REFERENCES}

1. Spanier BW, Tuynman HA, van der Hulst RW, Dijkgraaf MG, Bruno MJ. Acute pancreatitis and concomitant use of pancreatitis-associated drugs. Am J Gastroenterol 2011;106:2183-2188.

2. Kim YS, Cho JH. Drug induced acute pancreatitis. Korean J Pancreas Biliary Tract 2017;22:153-157.

3. Gagnon AL, Lavoie A, Frigon MP, Michaud-Herbst A, Tremblay K. A drug-induced acute pancreatitis retrospective study. Can J Gastroenterol Hepatol 2020;2020: 
1516493.

4. Badalov N, Baradarian R, Iswara K, Li J, Steinberg W, Tenner S. Drug-induced acute pancreatitis: an evidence- based review. Clin Gastroenterol Hepatol 2007;5:648-

661; quiz 644. 